
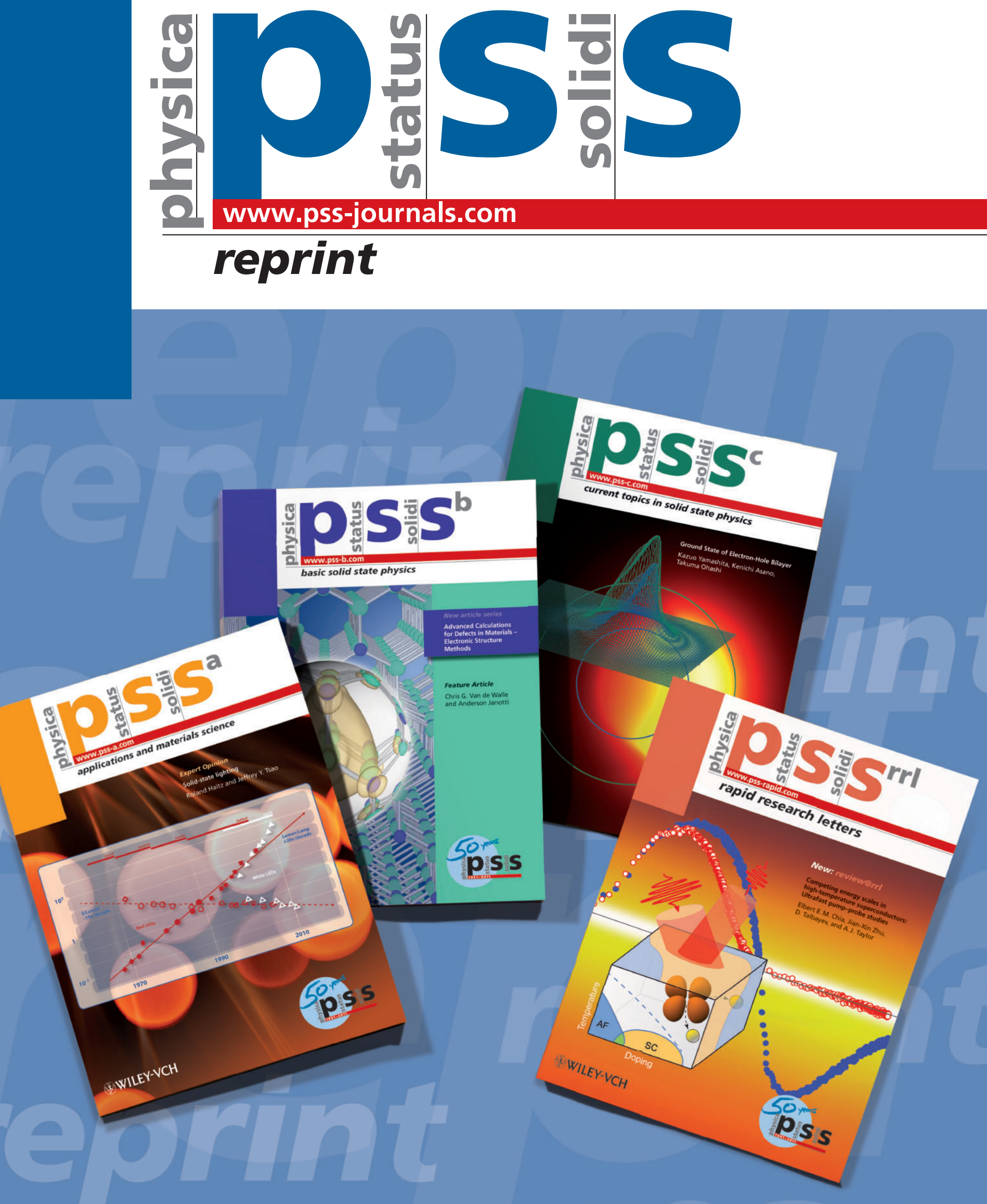


\title{
Free standing modulation doped core-shell GaAs/AIGaAs hetero-nanowires
}

\author{
Dance Spirkoska*, , Anna Fontcuberta i Morral ${ }^{1,2}$, Joseph Dufouleur', Qiushi Xie', \\ and Gerhard Abstreiter ${ }^{1,3}$ \\ ${ }^{1}$ Walter Schottky Institut and Physik Department, Technische Universität München, Am Coulombwall 4, 85748 Garching, Germany \\ ${ }^{2}$ Laboratoire des Matériaux Semiconducteurs, Institut des Matériaux, Ecole Polytechnique Fédérale de Lausanne, 1015 Lausanne, \\ Switzerland \\ ${ }^{3}$ Institut for Advanced Study, Technische Universität München, 85748 Garching, Germany
}

Received 11 July 2011, revised 1 August 2011, accepted 2 August 2011

Published online 5 August 2011

Keywords core-shell nanowires, doping, molecular beam epitaxy, GaAs, AlGaAs

* Corresponding author: e-mail spirkoska@wsi.tum.de, Phone: +498928911412, Fax: +498928912704

Modulation doped $\mathrm{AlGaAs} / \mathrm{GaAs}$ core-shell nanowire structures were grown by molecular beam epitaxy. A Si deltadoping was introduced in the AlGaAs shell around the $\{110\}$ facets of the GaAs core. The wires are typically highly resistive at low temperatures. However, they show a pronounced persistent photoconductivity effect indicating activation of free carriers from the delta-doped shell to the GaAs core. The n-type character of the channel is demonstrated by applying a back-gate voltage.
1 Introduction The invention of modulation or selective doping $[1,2]$ in planar semiconductor heterostructures has led to an increase of the electron mobilities $\mu$ at low temperatures by orders of magnitude during the last three decades [3]. Two-dimensional electron systems (2DES) formed by this means in $\mathrm{AlGaAs} / \mathrm{GaAs}$ heterojunctions have been the basis for many important discoveries in, both, fundamental physics and applied science such as the fractional quantum Hall effect [4] and the high electron mobility transistor, respectively. More recently, semiconductor nanowires have attracted great interest since they offer enhanced functionality in various areas such as nanoelectronics, optoelectronics, biosensing and energy harvesting [5]. If nanowires are combined with modulation doping further perspectives in nanoelectronics might be possible. Recently, a first report on modulation doping in core-shell nanowires buried in a GaAs matrix was published [6] with indications of the formation of a one-dimensional (1D) electron channel. However, only two terminal differential resistance was measured and gate control of the carrier density was not possible.

In this work, we report on first successful attempts to combine modulation doping with free standing radial het- ero-nanowires. We have fabricated GaAs nanowires in a catalyst-free molecular beam epitaxy (MBE) process combined with a remotely doped $\mathrm{AlGaAs} / \mathrm{GaAs}$ heterojunction grown as a core-shell structure using the method described in Ref. [7].

2 Results An illustration of the cross-section of the intended structure is presented in Fig. 1(a). The GaAs core is overgrown with an AlGaAs shell. A $\delta$-doped Si layer is inserted at a certain distance from the core. The whole structure is covered with a thin layer of GaAs to protect the AlGaAs shell. Electrons are expected to accumulate at the GaAs/AlGaAs interface leading to the formation of six planes with 2DES as well as 1D channels in the corners for thick enough cores [8]. The conduction band profile and the electron density along the red line in (a) calculated using the nextnano ${ }^{3}$ simulation package [9] for a core thickness of $100 \mathrm{~nm}$ are presented in Fig. 1(b). Experimentally, the GaAs nanowires were fabricated by the galliumassisted catalyst-free growth method in a high purity MBE system $[10,11]$. Two-inch (111)B GaAs wafers were coated with a sputtered $10 \mathrm{~nm}$ thick silicon dioxide film and used as substrates. The nanowire growth was carried 


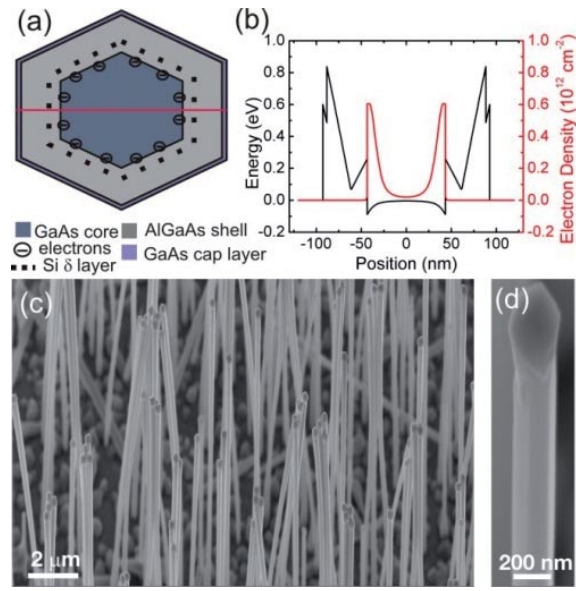

Figure 1 (online colour at: www.pss-rapid.com) (a) Schematic cross-section of the intended modulation doped core-shell nanowires. (b) Conduction band diagram and electron density along the red line in (a). (c) SEM micrograph from the grown core-shell nanowires. (d) Closer view of a single nanowire.

out at a nominal Ga flux of $0.25 \AA / \mathrm{s}, \mathrm{As}_{4}$ partial pressure of $2 \times 10^{-6}$ mbar (Ga-rich conditions), a substrate temperature of $630{ }^{\circ} \mathrm{C}$ and with a substrate rotation of $7 \mathrm{rpm}$. As we have previously shown, the nanowires grown under such conditions are oriented perpendicular to the substrate surface [12]. They are composed of nominally undoped GaAs, with pure zinc-blende structure and a low density of twins $[13,14]$. The nanowires reach a total length of $9 \mu \mathrm{m}$ and a typical diameter of $80-100 \mathrm{~nm}$ after 3 hours of growth. They have typically a hexagonal cross-section with the six side facets belonging to the $\{110\}$ crystal family [13]

Growth of high quality heterostructures on the "neutral" (110) surface is much more challenging compared to the typically used polar (001) surface [15]. Doping with Si requires also a careful choice of the growth parameters due to the amphoteric character of $\mathrm{Si}$ atoms in the GaAs crystal lattice [16]. In order to incorporate $\mathrm{Si}$ as donors on the $\mathrm{Ga}$ sites high As partial pressure and low substrate temperature are required [17]. It is for these reasons that high electron mobilities are hard to achieve on (110) surfaces. Only after accurate optimization of the growth conditions, record electron mobilities of $4.2 \times 10^{6} \mathrm{~cm}^{2} / \mathrm{Vs}$ have been reported at (110) $\mathrm{GaAs} / \mathrm{AlGaAs}$ interfaces for samples grown in the very same MBE system that we have used [18].

In order to perform the overgrowth of the nanowires we increased the As pressure up to $5 \times 10^{-5}$ mbar which results in a crystallization of the Ga droplet, and termination of the growth of the nanowires in the vertical direction. For MBE growth on the side facets we have reduced the substrate temperature to $465{ }^{\circ} \mathrm{C}$ and kept the $\mathrm{Ga}$ growth rate $(0.25 \AA / \mathrm{s})$ constant. We have shown in our previous work [19] using high-resolution transmission electron microscopy and photoluminescence spectroscopy that these growth parameters result in high quality and homogeneous core-shell structures. The shell thickness, however, differs from the nominal thickness due to the substrate rotation in- duced geometrical effects. Thus the growth times have to be scaled accordingly. We were aiming at a $90 \mathrm{~nm}$ thick $\mathrm{Al}_{0.4} \mathrm{Ga}_{0.6} \mathrm{As}$ shell, capped with $5 \mathrm{~nm}$ of GaAs. A Si delta doped layer was introduced in the $\mathrm{Al}_{0.4} \mathrm{Ga}_{0.6} \mathrm{As}$ shell at a distance of approximately $20 \mathrm{~nm}$ from the core interface by stopping the AlGaAs growth for $270 \mathrm{~s}$. The growth time of the $\mathrm{Si}$ source was adjusted accordingly in order to obtain an electron density in the low $10^{11} \mathrm{~cm}^{-2}$ range. An SEM micrograph of part of the grown nanowires is presented in Fig. 1(c), while in Fig. 1(d) a single core-shell heterostructure nanowire is shown. The crystallized Ga droplet is visible at the tip of the nanowire. Furthermore, negligible tapering is observed indicating quite homogeneous wire and shell growth. The Al content in the shell is about $40 \%$ as determined from Raman measurements on the most homogeneous samples which agrees well with the nominal Al content. In order to get more information about the quality of the heterostructures we have also grown samples on planar (110) oriented GaAs substrates using the same growth conditions. 2D carrier densities of $2 \times 10^{11} \mathrm{~cm}^{-2}$ were obtained at liquid He temperatures after illumination (persistent photoconductivity) with highest mobilities of about $300000 \mathrm{~cm}^{-2} / \mathrm{Vs}$ for a spacer layer thickness of $80 \mathrm{~nm}$. However the results obtained with such planar reference samples cannot be directly correlated with the nanowire overgrowth as the growth is much slower and more complex on the narrow facets and the twinning defects of the core nanowires can additionally influence the growth quality.

We have fabricated single-nanowire field effect transistor (FET) devices in a planar geometry on a heavily doped and oxidized $\mathrm{Si}$ substrate which acts as a global back gate. The top source and drain contacts were defined by electron beam lithography. Ohmic contacts on the nanowires were achieved by carefully cleaning the nanowire surface and subsequent electron beam evaporation of a sequence of metal layers consisting of $\mathrm{AuGe} / \mathrm{Ni} / \mathrm{Ti} / \mathrm{Au}(50 / 20 / 100 / 190 \mathrm{~nm})$ followed by a rapid thermal annealing for $20 \mathrm{~s}$ at $360{ }^{\circ} \mathrm{C}$. We have found that, relatively wide contacts $(2-3 \mu \mathrm{m})$ on the nanowire are necessary for reasonably low contact resistances. Given the length of our nanowire, we therefore could integrate maximal three contacts. The geometrical distance between the contacts is $1 \mu \mathrm{m}$. A single nanowire FET device is shown schematically in Fig. 2(a). An optical microscope

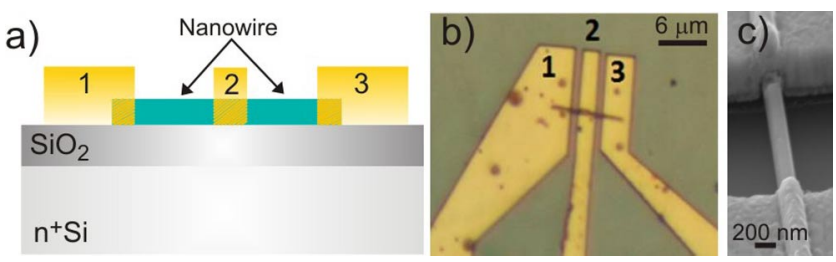

Figure 2 (online colour at: www.pss-rapid.com) (a) Schematic presentation of the nanowire FET device. (b) Optical microscope image of the investigated sample. (c) SEM micrograph of a typical contacted nanowire. 

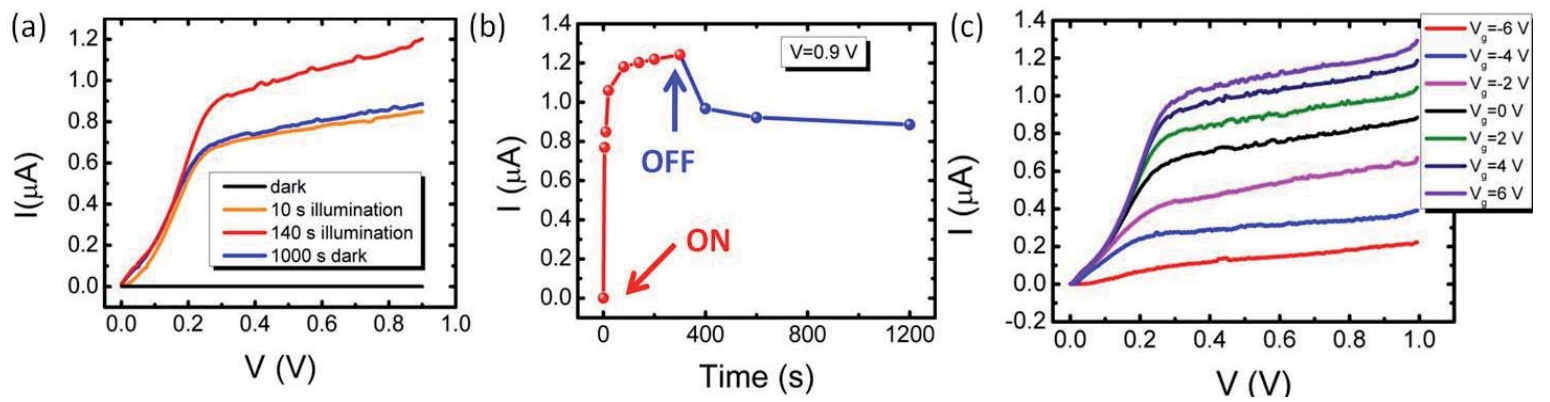

Figure 3 (a) $I-V$ characteristics recorded between contact 1 and 2. (b) Time dependence of the current at $V=0.9 \mathrm{~V}$ for different illumination times and in the dark. (c) $I-V$ characteristics recorded for different applied back gate voltages.

image of the sample discussed later in the text is presented in Fig. 2(b), while a SEM micrograph from part of one fabricated device is presented in Fig. 2(c). 2-point measurements were performed between contacts 1 and 2 , while for 3-point measurements the current was applied between contacts 1 and 3 , and the voltage was measured between 1 and 2. Typical resistances measured from more than 20 nanowire FET devices at room temperature are in the range of a few $100 \mathrm{k} \Omega$. Due to the measurement configuration the measured resistances include also the contact resistance. All investigated samples show insulating behavior after cooling down to $4.2 \mathrm{~K}$ in the dark (black line in Fig. 3(a)). However, a finite resistance is regained after illumination with a red LED. The $I-V$ characteristics show first a steep increase of the current with increasing DC bias voltage and nearly a saturation at about $1 \mu \mathrm{A}$ for voltages larger than $0.3 \mathrm{~V}$. Typical DC $I-V$ traces measured between contacts 1 and 2 for different illumination times are shown in Fig. 3(a). After switching off the illumination the device remains conducting with a slightly decreased value of saturation current. The photoconductivity measured as a function of illumination time is shown in Fig. 3(b) for an applied source-drain voltage of $0.9 \mathrm{~V}$. The current shows first a steep increase and saturates at about $1.25 \mu \mathrm{A}$ for $200 \mathrm{~s}$ of constant illumination. After switching off the LED the current first decreases and then reaches a constant value at about $0.85 \mu \mathrm{A}$. Such a persistent photoconductivity is known from modulation-doped planar heterostructures and is related to so-called DX centers which form deep levels in $\mathrm{Al}_{x} \mathrm{Ga}_{1-x} \mathrm{As}$ for $0.20<x<0.40$ [20]. It is also observed in our planar samples. The persistent photoconductivity is significantly larger in the nanowires indicating a higher density of deep levels in the shell structure. A more detailed analysis of the $\mathrm{d} I / \mathrm{d} V$ characteristics at low voltages between contacts 1,2 , and 3 reveals that about $80 \%$ of the measured resistance is due to the contacts. From this we estimate the resistance of the nanowire to be about $50 \mathrm{k} \Omega$. Taking the geometry into account with a 2DES at each of the six facets with a width of $50 \mathrm{~nm}$ and a length of the wire of $1 \mu \mathrm{m}$ between the contacts we can estimate the resistivity to be about $15 \mathrm{k} \Omega$. Applying a global back-gate voltage results in a typical transistor characteristics where the source-drain current is reduced for negative and increased for positive gate voltages, respectively
(Fig. 3(c)). This confirms that an n-channel is formed in the nanowire, as expected for the used growth parameters. This n-type behavior was observed in all fabricated FET devices.

3 Conclusion We have fabricated free standing modulation doped $\mathrm{AlGaAs} / \mathrm{GaAs}$ core-shell nanowire structures by MBE and analyzed the electrical properties by low-temperature photoconductivity experiments. The persistent photoconductivity and the gate voltage dependences demonstrate the formation of electron channels in the nanowires and opens new possibilities for modulation doped nanowire FETs.

Acknowledgements We thank Max Bichler for the expert experimental help with the MBE machine, Ilaria Zardo and Stefan Funk for Raman measurements and Stefan Birner for the help he provided with the nextnano ${ }^{3}$ software. The financial support by the DFG via the excellence cluster "Nanosystems Initiative Munich" and the collaborative research center SFB 631 as well as by EU via the Marie Curie Excellence Grant "SENFED" and the SNF (121758) is greatly acknowledged.

\section{References}

[1] H. L. Stormer et al., Solid State Commun. 29, 705 (1979).

[2] G. Abstreiter et al., Phys. Rev. Lett. 42, 1308 (1979).

[3] L. Pfeiffer et al., Appl. Phys. Lett. 55, 1888 (1989).

[4] D. C. Tsui et al., Phys. Rev. Lett. 48, 1559 (1982).

[5] C. M. Lieber et al., MRS Bull. 32, 99 (2007).

[6] D. Lucot et al., Appl. Phys. Lett. 98, 142114 (2011).

[7] A. Fontcuberta i Morral et al., Small 4, 899 (2008).

[8] G. Ferrari et al., Nano Lett. 9, 1631(2009).

[9] S. Birner et al., IEEE Trans. Electron Devices 54, 2137 (2007).

[10] C. Colombo et al., Phys. Rev. B 77, 155326 (2008).

[11] D. Spirkoska et al., J. Phys.: Condens. Matter 20, 454225 (2008).

[12] A. Fontcuberta i Morral et al., Appl. Phys. Lett. 92, 063112 (2008).

[13] D. Spirkoska et al., Phys. Rev. B 80, 245325 (2009).

[14] I. Zardo et al., Phys. Rev. B 80, 245324 (2009).

[15] P. Tejedor et al., Phys. Rev. B 59, 2341 (1999).

[16] D. Sun et al., J. Cryst. Growth 132, 166 (1993).

[17] F. Fischer et al., Appl. Phys. Lett. 86, 192106 (2005).

[18] F. Fischer et al., Physica E 22, 108 (2004).

[19] M. Heigoldt et al., J. Mater. Chem. 19, 840 (2009).

[20] E. F. Schubert et al., Phys. Rev. B 30, 7021(1984). 